\title{
Adaptive Dual-Rank Transmission Algorithm of SU-MIMO System Based on CSI Report Enhancement Mechanism
}

\author{
Xinhong Pan, Yafeng Wang \\ Wireless Theory \& Technology lab (WT\&T), Beijing University of Posts and Telecommunications \\ Beijing, China
}

Wireless Theory \& Technology lab (WT\&T), Beijing University of Posts and Telecommunications Beijing, China

pxh@bupt.edu.cn, wangyf@bupt.edu.cn

Keywords: SU-MIMO; CSI report enhancement; adaptive dual-rank transmission.

Abstract: In this paper, we proposed an adaptive dual-rank transmission algorithm underlying single-user MIMO (SU-MIMO) system. In SU-MIMO system, the rank indication (RI) is the main constraint to optimize the performance of the overall system. We employ the channel state information (CSI) report enhancement mechanism for modeling, and put forward reasonable formulas for constructing an auxiliary matrix which enable us to generate 2-port CSI reference signal (CSI-RS) and codeword, and calculating precoding matrix indicator (PMI) and codeword. And from the simulation results, the system performance can be significantly improved by the proposed algorithm.

\section{Introduction}

In order to make the wireless cellular systems support high-speed packet data services and make users get fast, high-quality multimedia applications, multiple input multiple output (MIMO) technology has been widely studied. The base station of SU-MIMO use active antenna array (AAS antenna) ${ }^{[1]}$ to implement a plurality of beams ${ }^{[2]}$. SU-MIMO technology can divide each vertical antenna element into several ports, in order to improve the space utilization, increase cell coverage and improve system performance and spectrum efficiency ${ }^{[3]}$ without changing the existing size of the antenna. However, we can do further with SU-MIMO technology. With the single-rank transmission supported by traditional Eigenvalue Based Beamforming (EBB) algorithm is increasingly unable to meet the current needs, many researchers try to apply dual-rank transmission in SU-MIMO to optimize the system performance, but there still are short of feasible and widely accepted algorithms and schemes.

In this paper, we research an adaptive dual-rank transmission algorithm based on CSI report enhancement mechanism in many scenes underlying TDD SU-MIMO system ${ }^{[4]}$. To achieve CSI report enhancement, we make the mobile station report the enhanced CSI to the base station, which includes RI, CQI (channel quality indicator) and PMI. In this algorithm, we constructs an auxiliary precoding matrix to generate 2-port CSI-RS and finally generate single-rank codeword or dual-rank codeword adaptively, and we select dual-rank transmission by generating dual-rank codeword when CSI report that channel is in good condition and therefore the system can send two signals each time and obtain better performance. Simulation results show that the SU-MIMO system that adopts adaptive dual-rank transmission algorithm can obtain better performance than general SU-MIMO system that adopts traditional EBB algorithm.

The rest of this paper is organized as follows. In section II, we present the system model and channel formulation. In section III, the adaptive dual-rank transmission algorithm based on CSI report enhancement mechanism is proposed. Simulation results are given in section IV. Finally, we summarize the conclusions in section V. 


\section{System Model}

In this paper, we assume there are $M$ transmit antennas and 2 receive antennas so we can achieve at most dual-rank transmission. And we implement CSI report enhancement mechanism ${ }^{[5,6]}$ by making BS transmit CSI-RS to MS and MS report enhanced CSI to BS. In this system model, MS transmits sounding reference signal (SRS) firstly, and then BS send CSI-RS to MS. After receiving CSI-RS, MS calculates and reports enhanced CSI to BS. BS generates and transmits codeword and other message. Finally MS generates retransmission message according to demodulation results. The system works as Fig.1.

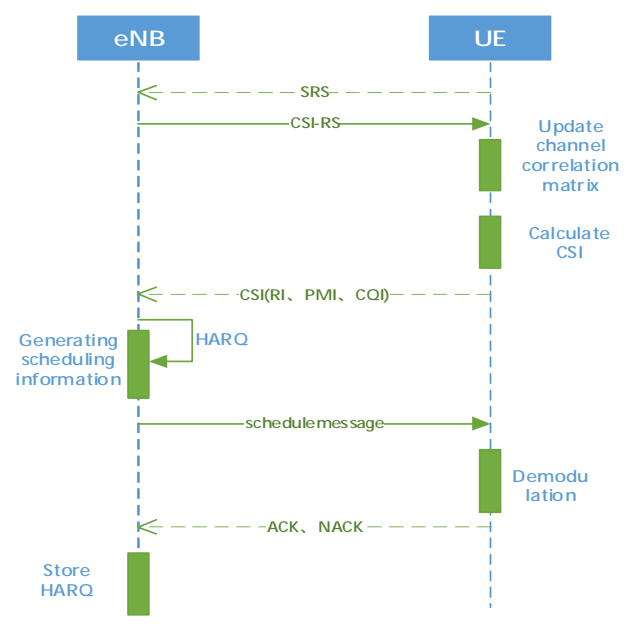

Fig. 1 Flow chart

The system channels used is ITU UMa, UMi from TR $36.873^{[7]}$ channel model. And compared with the general channel model ${ }^{[8,9]}$, the channel model used in this paper also uses the elevation arrival angle (ZOA) and elevation departure angle (ZOD). The channel between cluster $n$ and receive antenna element pair $u, s$ can be calculated as (1).

$$
\begin{aligned}
& \left.H_{u, s, n}(t)=\sqrt{P_{n} / M} \sum_{m=1}^{M}\left[\begin{array}{l}
\boldsymbol{F}_{r x, u, \theta}\left(\theta_{n, m, Z O A}, \varphi_{n, m, A O A}\right) \\
\boldsymbol{F}_{r x, u, \varphi}\left(\theta_{n, m, Z O A}, \varphi_{n, m, A O A}\right)
\end{array}\right]\right]^{T}\left[\begin{array}{cc}
\exp \left(j \Phi_{n, m}^{\oplus \theta}\right) & \sqrt{\kappa_{n, m}^{-1}} \exp \left(j \Phi_{n, m}^{\theta \varphi}\right) \\
\sqrt{\kappa_{n, m}^{-1}} \exp \left(j \Phi_{n, m}^{\varphi \theta}\right) & \exp \left(j \Phi_{n, m}^{\varphi \varphi}\right)
\end{array}\right] \\
& {\left[\begin{array}{l}
\boldsymbol{F}_{t x, s, \theta}\left(\theta_{n, m, Z O D}, \varphi_{n, m, A O D}\right) \\
\boldsymbol{F}_{t x, s, \varphi}\left(\theta_{n, m, Z O D}, \varphi_{n, m, A O D}\right)
\end{array}\right] \exp \left(j 2 \pi \lambda_{0}^{-1}\left(\hat{\boldsymbol{r}}_{r x, n, m}^{T} \bar{d}_{r, \mu}\right)\right) \exp \left(j 2 \pi \lambda_{0}^{-1}\left(\hat{\boldsymbol{r}}_{t x, n, m}^{T} \bar{d}_{t x, s}\right)\right) \exp \left(j 2 \pi v_{n, m} t\right)}
\end{aligned}
$$

where $\boldsymbol{F}_{r x, u, \theta}, \boldsymbol{F}_{r x, u, \varphi}$ are receive antenna element $u$ field patterns in the direction of the spherical basis vectors, $\AA^{\$}, \$$ respectively, and $\boldsymbol{F}_{t x, s, \theta}, \boldsymbol{F}_{t x, s, \varphi}$ are the transmit antenna element $s$ field patterns in the direction of the spherical basis vectors, $\AA^{\$}, \$$ respectively. $v_{n, m}$ is Doppler shift. $\hat{\boldsymbol{r}}_{r x, n, m}$ denotes a spherical unit vector determined by azimuth arrival angle $\varphi_{n, m, A O A}$ and elevation arrival angle $\theta_{n, m, Z O A}$ and it can be obtained as (2).

$$
\hat{\boldsymbol{r}}_{r x, n, m}=\left[\begin{array}{c}
\sin \theta_{n, m, Z O A} \cos \varphi_{n, m, A O A} \\
\sin \theta_{n, m, Z O A} \sin \varphi_{n, m, A O A} \\
\cos \theta_{n, m, Z O A}
\end{array}\right]
$$

where $n$ denotes a cluster and $m$ denotes a ray within $n . \hat{\boldsymbol{r}}_{t x, n, m}$ is a spherical unit vectors determined by the azimuth departure angle $\varphi_{n, m, A O D}$ and the elevation departure angle $\theta_{n, m, Z O D}$ and it can be calculated as (3) 


$$
\hat{r}_{t x, n, m}=\left[\begin{array}{c}
\sin \theta_{n, m, Z O D} \cos \varphi_{n, m, A O D} \\
\sin \theta_{n, m, Z O D} \sin \varphi_{n, m, A O D} \\
\cos \theta_{n, m, Z O D}
\end{array}\right]
$$

where $\overline{\boldsymbol{d}}_{x, u}$ denotes location vector of the receive antenna element $\mathrm{u}$, and $\overline{\boldsymbol{d}}_{t x, s}$ denotes location vector of the transmit antenna element $\mathrm{s} . \kappa_{n, m}$ is cross polarization power ratio. $\lambda_{0}$ is carrier wavelength.

\section{The algorithm description}

In this algorithm, we make the qualified users use dual-rank transmission according to CSI and thus improve packet data throughput and optimize system performance. And in order to support dual-rank transmission at most, we map $M$ transmit antennas into 2 ports. This algorithm focuses on mathematical implementation of how to construct precoding matrix, how to get the RI, PMI and how to generate the codeword. The algorithm related to codebook and CQI can refer to the Evaluation Methodology Document ${ }^{[10]}$ and not in this description. In this algorithm, we assume there are $n$ sub-bands (SB) in the whole band, $\boldsymbol{H}_{l i j}\left(i=1,2 \ldots n, j=1,2 \ldots n_{s}\right)$ is the subcarrier channel matrix $\mathrm{j}$ in SB $\mathrm{i}$ and it's a $2 \times M$ matrix. The calculation of each element in channel matrix refers to (1). $n_{s}$ is total number of subcarriers in each SB. We first construct an $M \times 2$ precoding matrix $\boldsymbol{W}_{i}(i=1,2 \ldots n)$ for SB i which can generates CSI-RS by combining with RS, and formulas is given by (4).

$$
\boldsymbol{W}_{i}=\left(\begin{array}{cc}
a_{1} & 0 \\
0 & a_{2} \\
a_{3} & 0 \\
\cdot & \cdot \\
\cdot & \cdot \\
\cdot & \cdot \\
0 & a_{M}
\end{array}\right) \quad\left(\boldsymbol{C}_{l i}=\frac{1}{S} \sum_{n=1}^{S} \boldsymbol{H}_{2 i j}{ }^{H} \boldsymbol{H}_{2 i j}=\boldsymbol{U}_{l i} \boldsymbol{A}_{l i} \boldsymbol{V}_{l i}{ }^{T}\right)
$$

where $\boldsymbol{C}_{1 \mathrm{i}}(i=1,2 \ldots n)$ is channel correlation matrix of SB $i$ and it's the average of all subcarriers channel correlation matrices in this SB. $S$ is total number of the subcarriers in SB $i . \boldsymbol{H}_{2 i j}\left(j=1,2 \ldots n_{s}\right)$ is the first row vector of $\boldsymbol{H}_{l i j}$ and it's a $M \times 1$ matrix.. $\boldsymbol{V}_{l i}$ is a result of SVD for $\boldsymbol{C}_{l i} .\left(a_{1}, a_{2} \ldots a_{64}\right)$ is the first column vector of $\boldsymbol{V}_{l i}$. $\boldsymbol{W}_{i}$ is generated by setting $\boldsymbol{T}$ as $\boldsymbol{W}_{i}$ 's first and second column vector and then replacing odd and even lines vector with 0 respectively. Then we can calculate channel correlation matrix as (5).

$$
\boldsymbol{C}_{2}=\frac{1}{N} \sum_{n=1}^{N}\left(\boldsymbol{W}_{i} \boldsymbol{H}_{l i j}\right)^{H}\left(\boldsymbol{W}_{i} \boldsymbol{H}_{l i j}\right)
$$

where $\boldsymbol{C}_{2}$ is channel correlation matrix of whole band and it's the average of the channel correlation matrices of all subcarriers. The subcarriers in SB $i$ use the same $\boldsymbol{W}_{i} . \boldsymbol{H}_{i j}\left(j=1,2 \ldots n_{s}\right)$ is subcarrier channel matrix $j$ in SB $i$. And then the best rank can be obtained by (6)

$$
R=\left\{\begin{array}{ll}
1 & \frac{\lambda_{1}}{\lambda_{2}} \leq 3 d B \\
2 & \frac{\lambda_{1}}{\lambda_{2}}>3 d B
\end{array} \quad\left(C_{2}=U_{2} \Lambda_{2} V_{2}^{T}\right)\right.
$$

where $R$ is the RI. $\lambda_{1}, \lambda_{2}$ denote the first and second eigenvalues of $\lambda_{2}$ which is one of the results of SVD for $C_{2} . P^{\prime} I_{i}(i=1,2 \ldots n)$ is precoding matrix indicator for SB $i$ and it can be calculated as (7). 


$$
P M I_{i}=k=\arg \max \left(\frac{1}{n_{s}} \sum_{p=1}^{n_{s}} \sum_{q=1}^{s}\left\|\left(\boldsymbol{H}_{l i p} \boldsymbol{W}_{i} \boldsymbol{B}_{R, k}\right)_{q}\right\|^{2}\right) \quad(k=0,1 \ldots \ldots \text { num })
$$

where $P M I_{i}$ is precoding matrix indicator, and $k$ is the codeword number. $\boldsymbol{B}$ is precoder codebook ${ }^{[12 \text {, }}$ ${ }^{13]}$ which can be selected according to $R$, and $\boldsymbol{B}_{R, k}$ is the codeword whose index is i in the precoder codebook corresponding to $R$. In this algorithm we use single-rank or dual-rank precoder codebook adaptively $\boldsymbol{H}_{\text {Iip }}$ is the subcarrier channel matrix $\mathrm{m}$ in SB i. $\mathrm{s}$ is total number of elements of the matrix $\boldsymbol{B}_{R, k} \boldsymbol{H}_{1 i p} \boldsymbol{W}_{i}$. num is total number of codewords of the precoder codebook.. And finally we can generate codeword $\boldsymbol{D}_{i}$ for SB i according to $P M I_{i}$, given by.

$$
\boldsymbol{D}_{i}=\boldsymbol{W}_{i}^{\prime} \boldsymbol{B}_{R, P M I_{i}}
$$

where $\boldsymbol{B}_{R, P M I_{i}}$ denotes a codeword ${ }^{[11,12]}$ whose number is equal to PMI in the precoder codebook corresponding to $R$. $W_{i}^{\prime}$ denotes a $M \times 2$ precoding matrix and its calculation process is similar to (5), (6), (7). The difference between $\boldsymbol{W}_{i}$ and $\boldsymbol{W}_{i}^{\prime}$ is the factor $\boldsymbol{H}_{2 i j}$. Because the channel changes at any time and there is a delay between CSI's generation in MS and CSI's arrival at BS, we choose the real-time channel matrix in the calculation of $\boldsymbol{W}_{i}$ and $\boldsymbol{W}_{i}^{\prime}$. If $R=2$, then $\boldsymbol{D}_{i}$ is a dual-rank codeword and it's a $M \times 2$ matrix; otherwise $\boldsymbol{D}_{i}$ is a single-rank codeword and it's a $M \times 1$ matrix.

The flow chart of the algorithm is shown in Fig.2.

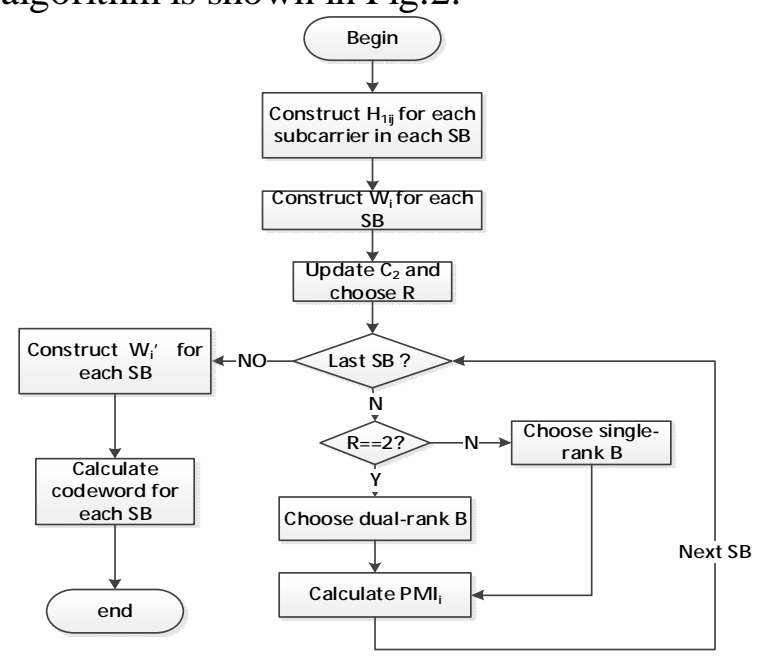

Fig. 2 Algorithm flow char

\section{Simulation Results}

The parameters are listed as Table I for a single-cell system:

TABLE I Simulation Parameters

\begin{tabular}{l|l}
\hline Parameters & Value \\
\hline Overhead boundaries & $3(\mathrm{~dB})$ \\
System bandwidth & $10(\mathrm{MHz})$ \\
Carrier frequency & $2 \mathrm{G}(\mathrm{Hz})$ \\
Under dip & $100($ degree $)$ \\
Operating mode & Synchronized \\
UE speed & $3(\mathrm{~km} / \mathrm{h})$ \\
Number of UE per BTS & 20 \\
Duplex mode & TDD \\
Distribution & Uniform \\
Number of users pairing & 1 \\
\hline
\end{tabular}


In this paper, the service model is FTP and the scenes are UMa and UMi. The baseline for comparison is traditional Eigenvalue Based Beamforming (EBB) algorithm. Numerical results of the proposed algorithms and EBB algorithm are presented below, and the main indicators in this simulation are 5\% (edge) user packet throughput (UPT), mean UPT and RU.

Fig. 3 shows that in UMa scene, the adaptive dual-rank transmission algorithm can improve edge and mean UPT by $28.65 \%$ and $31.57 \%$, respectively. Moreover, RU is reduced by $9.19 \%$. Fig. 4 shows that with the growth of proportion, the dual-rank transmission algorithm improves UPT faster. Fig. 3, 4 can prove that with the growth of rank, dual-rank transmission algorithm can improve system performance in UMa scene.

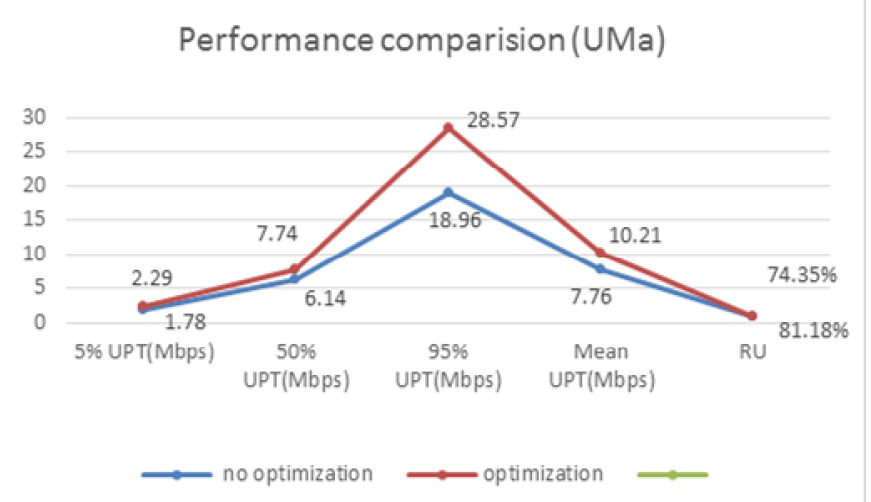

Fig. 3 Performance comparison (UMa)

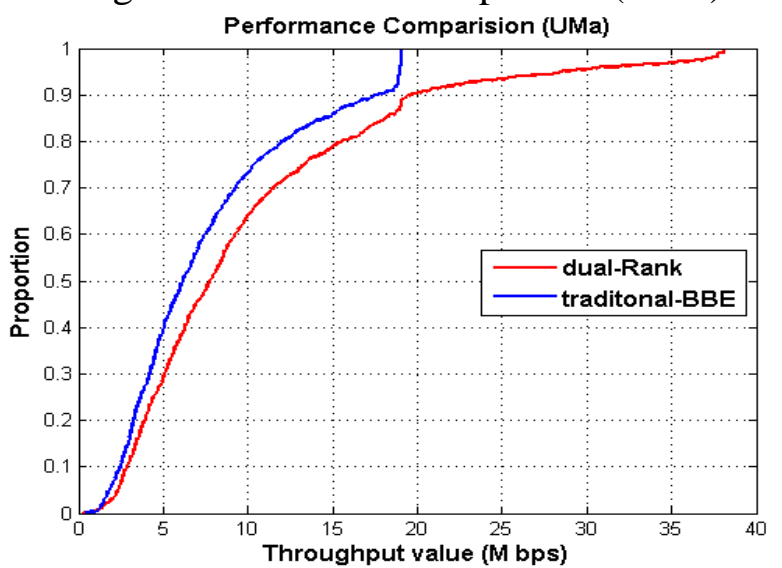

Fig. 4 Throughput (UMa)

As Fig.5 shows, in UMi scene, the adaptive dual-rank transmission algorithm can improve edge and mean UPT by $51.76 \%$ and $46.11 \%$, respectively. Moreover, RU is reduced by $24.67 \%$ while adopting dual-rank transmission algorithm. As Fig. 6 shows, with the growth of proportion, the dual-rank transmission algorithm improves UPT obviously faster. Fig 5, 6 can prove that with the growth of rank, dual-rank transmission algorithm can improve system performance in UMi scene.

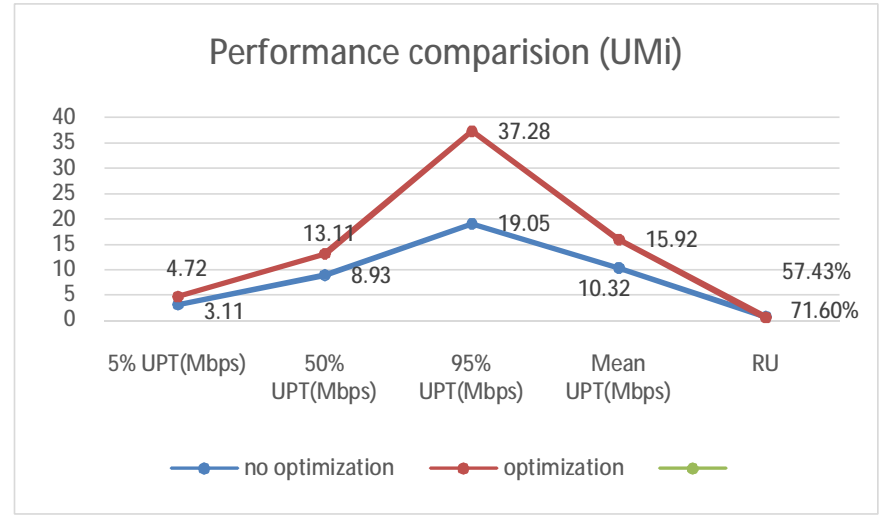

Fig. 5 Performance comparison (UMi) 


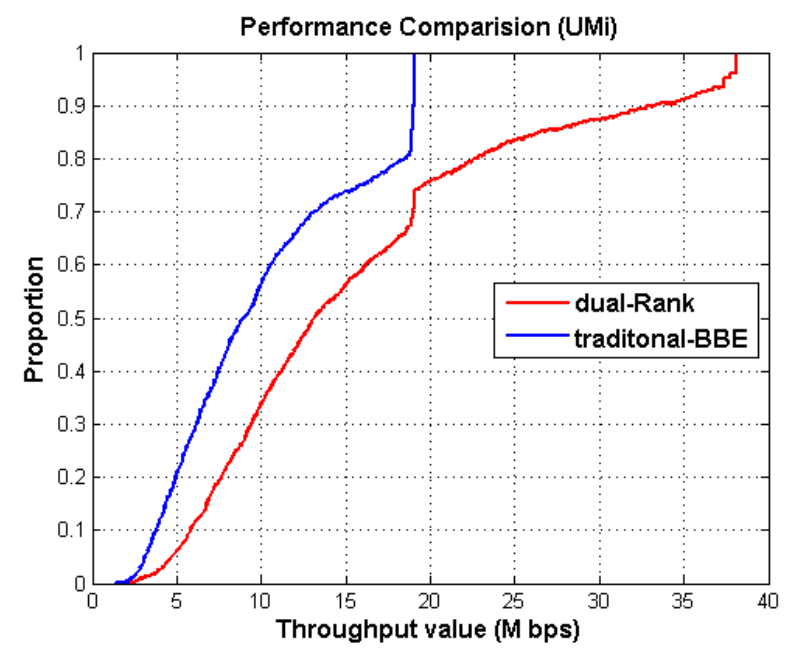

Fig.6 Throughput (UMi)

\section{Summary}

In this paper, we study adaptive dual-rank transmission algorithm of SU-MIMO system based on CSI report enhancement mechanism in many scenes underlying SU-MIMO system. In this algorithm, mobile station reports enhanced CSI and BS generated codeword based on precoding matrix and PMI. This algorithm can use single-rank and dual-rank codeword for transmission adaptively. Through simulation results, we demonstrate the feasibility and satisfied throughout and RU of proposed algorithms. In general, rational utilization of adaptive dual-rank transmission algorithm can optimize system performance in the SU-MIMO system.

\section{Acknowledgement}

This paper is sponsored by National Key Technology R\&D Program of China under grant No. 2014ZX03003011-004.

\section{References}

[1] G. Americas, "MIMO and Smart Antennas for 3G and 4G wireless systems," Whitepaper, pp.1-135, 2010.

[2] YAN Hua-liang. LTE-A Research channel modeling and 3D beamforming technology 3D MIMO [D]. Beijing University of Posts and Telecommunications, 2014.

[3] Yuwen Pan; Qinglin Luo; Guodong Li; Yan Zhao; Zhilan Xiong, "Performance Evaluation of 3D MIMO LTE-Advanced System," Vehicular Technology Conference (VTC Fall), 2013 IEEE 78th , vol., no., pp.1,5, 2-5 Sept. 2013.

[4] WANG Ying-min TD-LTE technology principle and system design Beijing: People's Posts and Telecommunications Press, 2010.

[5] 3GPP TSG-RAN WG1 \#80 R1-150448, "Feedback framework for diverse SU-MIMO antenna configurations," Athens, Greece, 9th - 13th February 2015.

[6] Lim C, Yoo T, Clerckx B, et al. Recent trend of multiuser MIMO in LTE-advanced [J]. Communications Magazine, IEEE, 2013, 51(3): 127-135.

[7] 3GPP TR 36.873,"3D channel model for LTE (Release 12)" , Tech. Rep. v1.0.0, Sep.2013.

[8] 3GPP TS 36.211, "E-UTRA; Physical Channels and Modulation (Release 11)," Tech. Rep. v 11.0.0, 2012.

[9] IST-4-027756, "WINNER II channel models," WINNER II, Tech. Rep. D1.1.2, v1.2, Feb. 2008.

[10] IEEE 802.16m-08/004r5 "Evaluation Methodology Document (EMD)," IEEE 802.16m 
2000-01-15.

[11]3GPP TS 36.213 " Table 7.2.4-1: Precoder codebook for 1-layer CSI reporting using antenna ports 15 to $22, " \mathrm{~V} 12.5 .02015-03$.

[12]3GPP TS 36.213 " Table 7.2.4-2: Precoder codebook for 2-layer CSI reporting using antenna ports 15 to $22, " \mathrm{~V} 12.5 .02015-03$. 